ARTICLE

\title{
Structure of the Bacillus subtilis 70 S ribosome reveals the basis for species-specific stalling
}

Daniel Sohmen', Shinobu Chiba ${ }^{2}$, Naomi Shimokawa-Chiba², C. Axel Innis ${ }^{3,4}$, Otto Berninghausen', Roland Beckmann ${ }^{1,5}$, Koreaki Ito ${ }^{2} \&$ Daniel N. Wilson ${ }^{1,5}$

Ribosomal stalling is used to regulate gene expression and can occur in a species-specific manner. Stalling during translation of the MifM leader peptide regulates expression of the downstream membrane protein biogenesis factor YidC2 (YqjG) in Bacillus subtilis, but not in Escherichia coli. In the absence of structures of Gram-positive bacterial ribosomes, a molecular basis for species-specific stalling has remained unclear. Here we present the structure of a Gram-positive B. subtilis MifM-stalled 70S ribosome at 3.5-3.9 $\AA$, revealing a network of interactions between MifM and the ribosomal tunnel, which stabilize a non-productive conformation of the PTC that prevents aminoacyl-tRNA accommodation and thereby induces translational arrest. Complementary genetic analyses identify a single amino acid within ribosomal protein $\mathrm{L} 22$ that dictates the species specificity of the stalling event. Such insights expand our understanding of how the synergism between the ribosome and the nascent chain is utilized to modulate the translatome in a species-specific manner.

\footnotetext{
${ }^{1}$ Gene Center and Department for Biochemistry, University of Munich, Feodor-Lynen-Street 25, Munich 81377, Germany. ${ }^{2}$ Faculty of Life Sciences, Kyoto Sangyo University, Motoyama, Kamigamo, Kita-Ku, Kyoto 603-8555, Japan. ${ }^{3}$ Institut Européen de Chimie et Biologie, Université de Bordeaux, Pessac, France. ${ }^{4}$ Institut National de la Santé et de la Recherche Médicale (U869), Bordeaux, France. ${ }^{5}$ Center for integrated Protein Science Munich (CiPSM), University of Munich, Feodor-Lynen-Street 25, Munich 81377, Germany. Correspondence and requests for materials should be addressed to D.N.W. (email: wilson@lmb.uni-muenchen.de).
} 
T he ribosome is the major protein-synthesizing machine in the cell that converts the genetic information present within the codons of the mRNA into an amino-acid sequence within the polypeptide chain ${ }^{1}$. Ribosomes can transiently pause or even become stalled for prolonged periods during the translation of mRNAs due to many intrinsic factors, such as secondary structure within the mRNA ${ }^{2}$ or the presence of particular amino-acid sequences within the nascent polypeptide chain, such as polyproline sequences ${ }^{3,4}$. In many cases, ribosome stalling occurs during translation of short upstream open reading frames, so-called leader peptides, which is utilized by both bacteria and eukaryotes to regulate expression of downstream genes $^{5,6}$. These regulatory events can be ligand-dependent, as exemplified by the ErmCL and TnaC leader peptides that require the presence of a macrolide antibiotic or free tryptophan, respectively, to induce ribosome stalling ${ }^{7,8}$, or ligand independent, with well-characterized examples including the E. coli SecM and B. subtilis MifM leader peptides, where translation of the amino-acid sequence is sufficient to induce the translational arrest ${ }^{9-11}$.

In B. subtilis, the MifM leader peptide is located upstream of the $y i d C 2(y q j G)$ gene (Fig. 1a), which cannot be translated independently of MifM because the ribosome-binding site of yidC2 is sequestered within a stem-loop structure in the mRNA (Fig. 1a) ${ }^{10}$. Ribosome stalling during translation of MifM leads to unwinding of the stem-loop structure and maintains the ribosome-binding site accessible for ribosome binding and induction of YidC2 expression (Fig. 1b). YidC2 is a homologue of the constitutively expressed SpoIIIJ (YidC1), a protein involved in membrane protein insertion and folding ${ }^{12}$. The $B$. subtilis MifM leader peptide is 95 amino-acids long and comprises a C-terminal region (residues 69-89) that is critical for ribosome stalling as well as an $\mathrm{N}$-terminal transmembrane (TM) segment that targets the MifM peptide for membrane insertion, presumably via SpoIIIJ ${ }^{10}$. Interaction between SpoIIIJ and the TM segment of MifM as it emerges from the ribosomal tunnel is thought to prevent ribosome stalling by providing a pulling force on the MifM nascent chain ${ }^{11}$, analogous to SecA relief of SecM stalling ${ }^{13-15}$. Subsequently, canonical translation termination and ribosome-recycling ensues, leading to rapid refolding of the mRNA and repression of YidC2 expression (Fig. 1a). In contrast, when cellular levels of SpoIIIJ are low, ribosome stalling occurs on MifM, maintaining the unfolded conformation of the mRNA and thereby promoting expression of YidC2. In this manner, $B$. subtilis ensures that sufficient levels of SpoIIIJ or YidC2 are present in the membrane to direct membrane protein insertion and/or protein folding ${ }^{10,16}$.

Biochemical studies have demonstrated that four major ribosome-stalling sites are present in the MifM leader peptide; the first occurring when the codon for residue D89 is present in the P-site, then ribosomes stall at the following three codon positions corresponding to residues A90, G91 and S92 (Fig. 1b) ${ }^{6}$. Mutagenesis studies have identified six residues (R69, I70, W73, I74, M80 and N81) as well as the negatively charged DEED sequence (residues 86-89) within the C-terminal region of MifM (Fig. 1b) that are important for ribosome stalling ${ }^{6,10}$. Despite the a

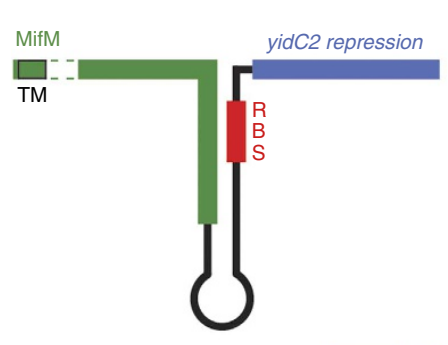

b

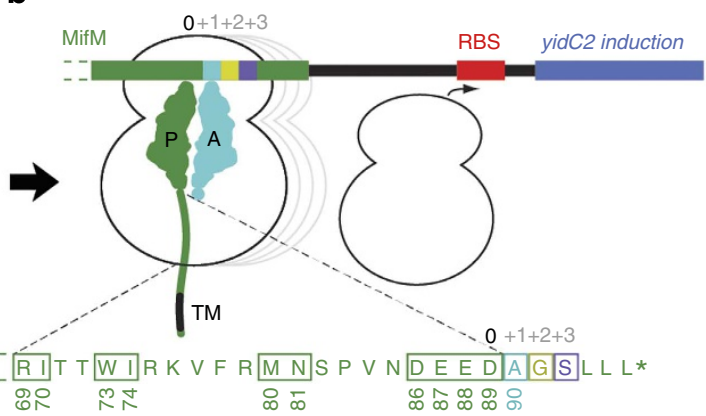

C

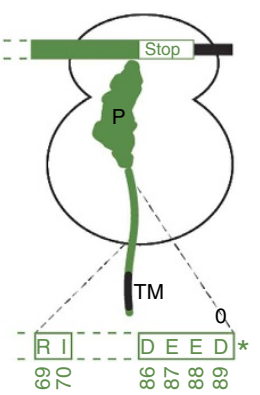

d

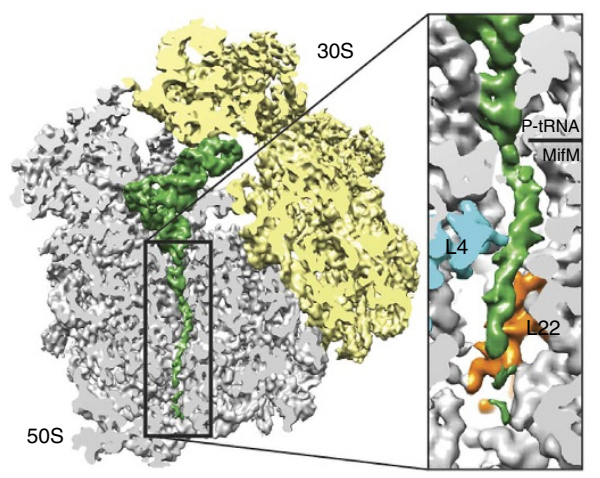

e

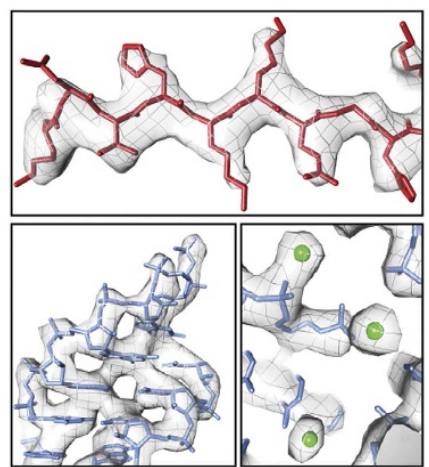

f

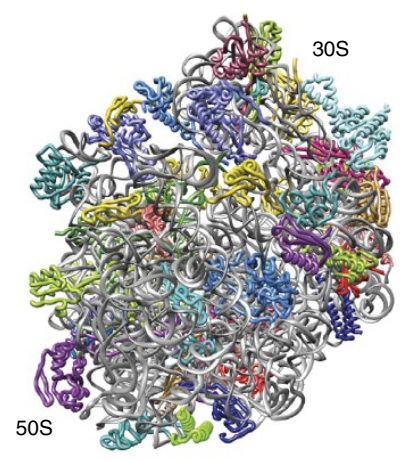

Figure 1 | Cryo-EM structure and molecular model of the $\mathbf{B}$. subtilis MifM-stalled ribosome complex. (a,b) Schematic of the mifM-yidC2 mRNA illustrating the N-terminal transmembrane (TM) segment (black, helix) and C-terminal stalling region (green) of the MifM leader peptide with the stemloop structure that sequesters the ribosome-binding site (RBS) of the yidC2 gene (blue). In (b) the multisite ribosome stalling $(0,+1,+2$ and +3$)$ during translation of MifM maintains the unfolded conformation of the mRNA allowing ribosome binding and induction of yidC2 expression. The MifM stalling sequence (residues 69-89) is shown with critical residues boxed in green. Asterisks indicate stop codons. (c) MifM-stalled ribosome complex used for cryo-EM. (d) Transverse section of the cryo-EM structure of the MifM-SRC (30S, yellow; 50S, grey) showing P-tRNA and MifM nascent chain (green) within the ribosomal tunnel and enlargement where ribosomal proteins L4 (cyan) and L22 (orange) are coloured. (e) Electron density (grey mesh) for selected regions of large subunit ribosomal protein and rRNA of the MifM-SRC. (f) Molecular model of the B. subtilis $70 \mathrm{~S}$ ribosome. 
high conservation of the ribosomal tunnel, translational stalling by MifM occurs on B. subtilis ribosomes, but not efficiently on $E$. coli ribosomes ${ }^{11}$; however, a molecular basis for this species specificity has remained unclear.

Here we have developed a $B$. subtilis cell-free in vitro translation system, which was subsequently used to generate a B. subtilis MifM-stalled ribosome complex (MifM-SRC). We present a cryoelectron microscopy (cryo-EM) reconstruction of the MifM-SRC at 3.5-3.9 $\AA$ resolution, which was used to build the first molecular model of a Gram-positive $70 \mathrm{~S}$ ribosome. The only contacts of the MifM nascent chain with the ribosome that are not conserved between B. subtilis and E. coli are located within the lower region of the exit tunnel where they involve ribosomal protein L22. Consistently, our complementary genetic mutagenesis studies identified a single amino-acid residue, Met90 of L22, which modulates the specificity of MifM-dependent stalling. Interaction of the MifM nascent chain within the ribosomal tunnel positions the sidechain of Glu88 of MifM in a manner that prevents accommodation of an incoming aminoacyltransfer RNA (tRNA) at the peptidyltransferase centre (PTC) of the ribosome, thus providing a structural basis for the MifMdependent translational arrest.

\section{Results}

Cryo-EM structure of B. subtilis MifM-SRC. To understand the structural basis for the mechanism of MifM-dependent translational stalling and provide structural insight into the species specificity of MifM stalling, we set out to structurally characterize the B. subtilis MifM-SRC. Unlike for E. coli, translation systems for $B$. subtilis are not commercially available. Therefore, to generate complexes of translating $B$. subtilis ribosomes, we developed and optimized a B. subtilis S12 lysate-based in vitro coupled transcription-translation system (Supplementary Fig. 1 and Methods). This system was used to prepare B. subtilis MifMSRCs by translation of a template encoding the B. subtilis MifM leader peptide (Supplementary Fig. 1 and Methods). Homogeneity of the MifM-SRC was increased by replacing Ala90 of MifM with a UAA stop codon (Supplementary Fig. 1), which arrests ribosomes at the first stall site (Fig. 1c), and thereby prevents further translation and subsequent arrest at downstream stall sites $^{6}$. Like the wildtype MifM-SRC, the MifM-Ala90UAA stalled ribosomes are refractory to the action of puromycin, and are also not subject to the action of the termination release factors RF1 and RF2 ${ }^{6,10}$. This MifM-SRC (Fig. 1c) was separated from non-translating ribosomes and polysomes using sucrose gradient centrifugation and affinity chromatography, as performed previously for other SRCs ${ }^{17,18}$. A single MifM-peptidyl-tRNA product, and no free MifM peptide, was detected in the final MifM-SRC (Supplementary Fig. 1), indicating the homogeneity of the sample and its suitability for structural analysis. A singleparticle reconstruction of the MifM-SRC was obtained from 305,045 particles using cryo-EM (Fig. 1d). The MifM-SRC has an average resolution of $3.9 \AA$, with local resolution calculations ${ }^{19}$ indicating that the resolution of the core of the 50S subunit reaches $3.5 \AA$ (Supplementary Fig. 2). This is consistent with the features of the cryo-EM map, such as strand separation in $\beta$-sheets, the pitch of $\alpha$-helices as well as density for many side chains of the ribosomal proteins (Fig. 1e). In addition, the rRNA backbone and nucleotides are well resolved, as well as the position of many putative magnesium ions (Fig. 1e). The distinct features of the electron density (Supplementary Figs 3 and 4), together with the high homology to E. coli and T. thermophilus $70 \mathrm{~S}$ ribosomal structures ${ }^{20,21}$, allowed a molecular model of the B. subtilis $70 \mathrm{~S}$ ribosome to be built (Fig. 1f, Table 1 and Supplementary Figs 5 and 6), encompassing a total of 4,579 of the

\section{Table 1 | Refinement and Model Statistics.}

\begin{tabular}{|c|c|}
\hline \multicolumn{2}{|l|}{ Data collection and refinement } \\
\hline Particles & 305,045 \\
\hline Pixel size $(\AA)$ & 1.108 \\
\hline Defocus range $(\mu \mathrm{m})$ & $1.0-2.5$ \\
\hline Voltage $(\mathrm{kV})$ & 300 \\
\hline Electron dose $\left(e^{-} / \AA^{-2}\right)$ & 28 \\
\hline Map sharpening $B$ factor $\left(\AA^{2}\right)$ & -124.35 \\
\hline Resolution ( $\AA$, 0.143 FSC) & 3.9 \\
\hline \multicolumn{2}{|l|}{ Model Composition } \\
\hline Non-hydrogen atoms & 135,413 \\
\hline Protein residues & 5660 \\
\hline RNA bases & 4675 \\
\hline \multicolumn{2}{|l|}{ R.m.s. deviations } \\
\hline Bonds $(\AA)$ & 0.0094 \\
\hline Angles $(\AA)$ & 1.46 \\
\hline \multicolumn{2}{|l|}{ Validation (proteins) } \\
\hline Molprobity score (79th percentile) & 1.94 \\
\hline Clashscore, all atoms (80th percentile) & 8.38 \\
\hline Good rotamers (\%) & 99.85 \\
\hline \multicolumn{2}{|l|}{ Ramachandran plot } \\
\hline Favored (\%) & 91.95 \\
\hline Outliers (\%) & 1.98 \\
\hline \multicolumn{2}{|l|}{ Validation (RNA) } \\
\hline Correct sugar puckers (\%) & 97.75 \\
\hline Good backbone conformation (\%) & 100 \\
\hline
\end{tabular}

4,602 (99.5\%) nucleotides of the 5S, $16 \mathrm{~S}$ and 23S rRNAs, 19 small subunit ribosomal proteins and 29 of the 32 large subunit proteins (L1, L7/L12 and L9 were excluded). The slightly lower resolution of the small subunit ( $4.0 \AA$, Supplementary Fig. 2) restricted the modelling of the small subunit ribosomal proteins to a backbone trace, whereas sidechains were modelled for the large subunit proteins.

The majority of the rRNA in the core of $B$. subtilis $70 \mathrm{~S}$ ribosome is structurally conserved with E. coli and T. thermophilus ribosomes, however, specific regions resemble only E. coli (h26, h33, h44 in the 30S; H27, H68 and H79 in the 50S) or T. thermophilus (h9 in the 30S; H15 and H63 in the 50S), whereas others appear to be Bacillus specific (h6, h10, h17 in the 30S; H25 and $\mathrm{H} 54$ in the 50S; Supplementary Fig. 8). As expected from the genome sequence, the $B$. subtilis 70 S ribosome lacks density for S21 (Supplementary Fig. 9), which is present in E. coli but absent in T. thermophilus. Surprisingly, density for L9 and L25 (also referred to as the general stress protein CTC) is also absent in the B. subtilis 70 S ribosome (Supplementary Fig. 9), despite L9 and L25 being encoded in the B. subtilis genome. Only one tRNA is present in the MifM-SRC, located at the P-site, where it makes codon-anticodon interactions with P-site codon of the mRNA (Fig. 2a,b). No density for the mRNA is observed in the A-site, nor within the downstream mRNA channel, whereas in contrast, the upstream $5^{\prime}$ end of the mRNA can be unambiguously traced from the P-site through the E-site to the back of $30 \mathrm{~S}$ subunit where it establishes a number of non-canonical base-pairs with the $3^{\prime}$ end of the 16S rRNA resulting in a 8-basepair helix (Fig. 2c-e), analogous to the Shine-Dalgarno (SD)-anti-SD helices observed during elongation ${ }^{22,23}$ (Fig. 2f). The CCA-end of the P-tRNA adopts its canonical position at the PTC of the ribosome, with clear density for the MifM nascent polypeptide chain extending from the ribose of A76 and traversing the entire 
a

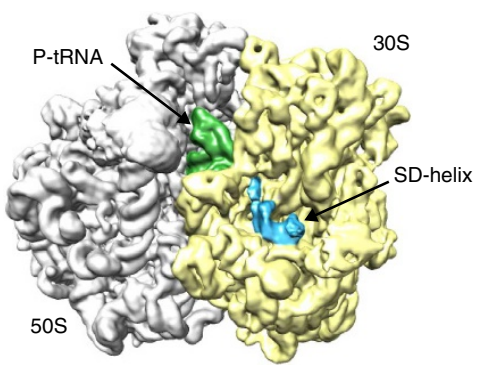

b

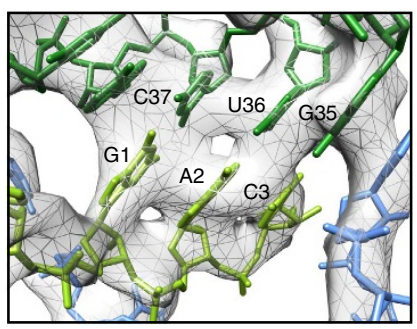

C

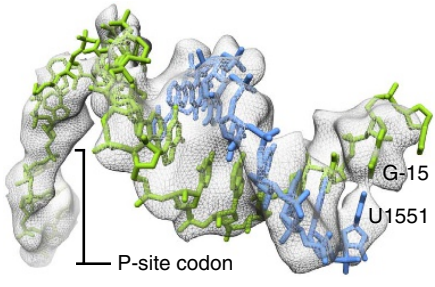

d
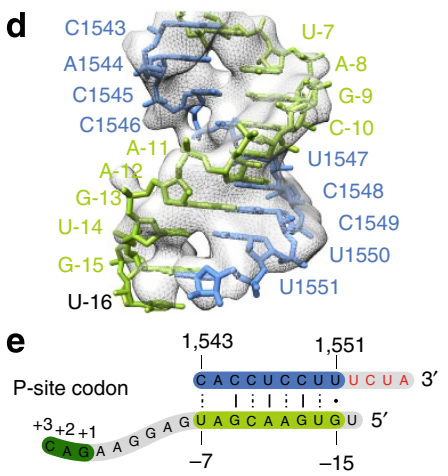

f

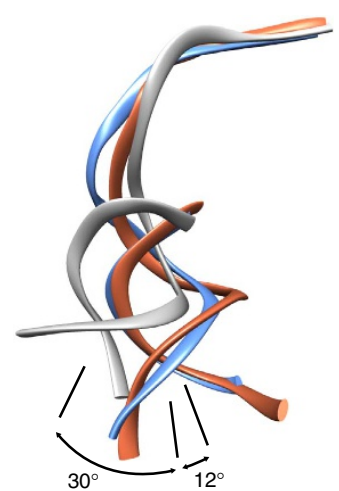

Figure 2 | The path of the mRNA through the B. subtilis MifM-SRC. (a) Location of Shine-Dalgarno(SD)-anti-SD-like helix on 70S ribosome (30S, yellow; 50S, grey, P-tRNA, green). (b) Codon-anticodon interaction between P-tRNA (dark green) and mRNA (pale green). (c) Electron density (grey mesh) and molecular model for the path of the mifM mRNA (green) from the P-site to back of the 30S subunit (d) where it forms an 8 base pair SD-anti-SD-like helix with the $3^{\prime}$ end of the 16S rRNA (blue). (e) Schematic for the non-canonical base-pairing observed in the SD-anti-SD-like helix of the MifM-SRC. (f) Comparison of SD-anti-SD-like helix observed in B. subtilis MifM-SRC (blue) with initiation SD-anti-SD helix (grey) and post-initiation SD-anti-SD helix (red) observed previously22.

length of the ribosomal exit tunnel (Fig. 1b). Local resolution calculations indicate that the MifM nascent chain exhibits some degree of flexibility (Supplementary Fig. 2), particularly in regions that do not appear to establish contacts with components of the ribosomal tunnel.

MifM interactions with the ribosome. In the upper region of the tunnel, the density for the MifM nascent chain is well resolved at the sites of contact, namely adjacent to the PTC and at the constriction, enabling side chains of MifM residues E87/D89 and R79-N81 to be modelled (Fig. 3a-c). In contrast, the MifM nascent chain becomes fragmented in the lower region of the tunnel, indicating a higher degree of flexibility, and was therefore filtered to $4 \AA$ to obtain continuous uninterrupted electron density (Fig. 3d-f). For the less resolved regions, that is, S82-E86 and W73-F78, we present a model for the backbone trace and acknowledge that the register for MifM may become lost after the constriction in the lower regions of the tunnel. The model localizes the three pairs of critical MifM residues, M80-N81, W73-I74 and R69-I70, at the sites of contact with components of the ribosomal tunnel (Fig. 3b,f).

In addition to the PTC (discussed later), four main interactions are observed between MifM and the ribosome: Two contacts are observed at the constriction, which sandwich the MifM nascent chain between Arg66 of L4 on one side of the tunnel and A751 (E. coli numbering is used throughout the text) of the 23S rRNA on the other (Fig. 3a-c). The contact to A751 is one of the strongest fusions of density between the tunnel wall and MifM, and appears to involve the backbone in the vicinity of R79 of MifM (Fig. 3a), whereas Arg79 and Asn81 coordinate interaction with the sidechain of Arg66 of L4. Consistently, the N81A mutation that abrogates MifM-stalling ${ }^{6,10}$ would also abolish the potential hydrogen bond formed with Arg66 of L4. Two contacts are observed within the lower region of the tunnel, one between Phe85 of L22 and Ile74 of MifM (Fig. 3d) and another between
A1321 in $\mathrm{H} 50$ of the 23S rRNA in the vicinity of His68 of MifM (Fig. 3f). These contacts are likely to be important since insertions within L22 compromise MifM-dependent stalling, as do mutations of Trp73, Ile74 or His68 to alanine ${ }^{10}$. However, because the H68A only partially affected YidC2 induction using the mifM-yidC2' - lac $Z$ reporter ${ }^{10}$, the latter contact with A1321 may instead reflect the importance of the neighbouring MifM residues Arg69 and Ile70 for stalling ${ }^{10}$. While a fully extended backbone conformation for MifM in the lower tunnel region does not bring these residues into direct proximity of A1321, because of the limited resolution for this region of the nascent chain we cannot rule out that the side chain of Arg69 can reach down to directly contact A1321. In agreement with the observed contacts between MifM and the ribosome, we could demonstrate using a B. subtilis in vivo GFP-MifM-LacZ (translational fusion) reporter (Fig. 3g) that both L22 and L4, but not L23 (the only other ribosomal protein that contributes to the exit tunnel), are important for MifM stalling (Fig. $3 \mathrm{~h}-\mathrm{j}$ ): In a wildtype B. subtilis strain, translation of the GFP-MifM-LacZ reporter leads to stalling within the MifM sequence and therefore negligible $\beta$-galactosidase is produced (Fig. $3 \mathrm{~h}, \mathrm{j}$ ), whereas $B$. subtilis strains bearing internal deletions within the tunnel lumen regions of $\mathrm{L} 22$ and $\mathrm{L} 4$ lead to an increase in $\beta$-galactosidase activity, consistent with a reduction in MifM stalling. In contrast, $\beta$-galactosidase activities remained low when deletions were made in L23 (Fig. 3j), consistent with the absence of a direct interaction between MifM and L23 in the MifM-SRC.

Species specificity of MifM stalling. To understand the species specificity of MifM stalling, we analysed the sequence and structure conservation of the ribosomal tunnel between $B$. subtilis and $E$. coli, revealing that the rRNA components are highly conserved, including nucleotides A751 and A1321 (Fig. 4a) and, similarly, the luminal region of L4 is invariant between $B$. subtilis and E. coli (Fig. 4b). In contrast, the $\beta$-hairpin of L22 exhibits a 
a

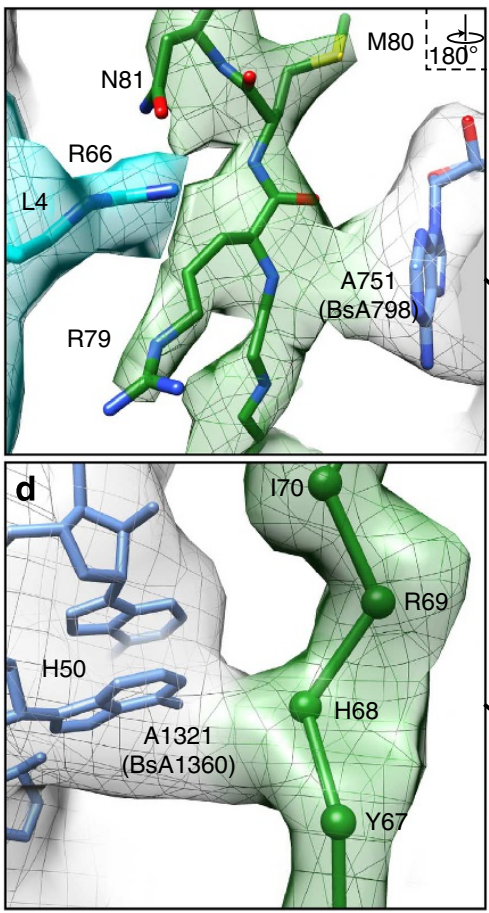

b

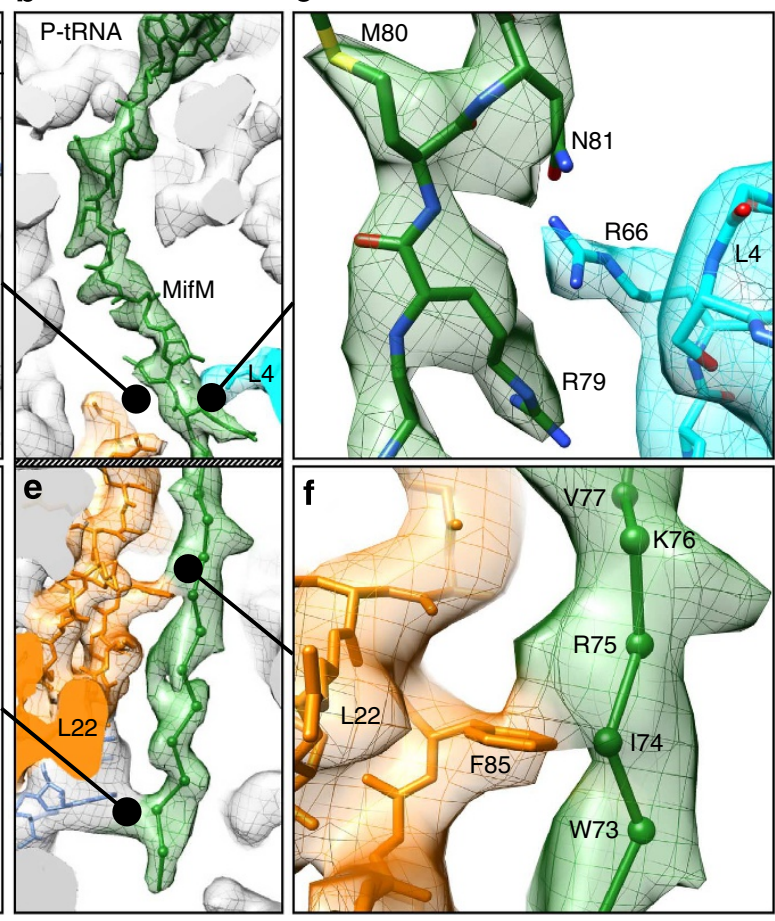

g

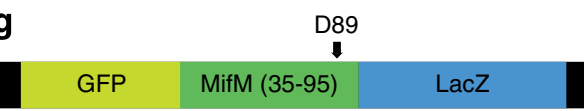

Translation arrest $\uparrow \beta$-galactosidase activity $\downarrow$

Translation arrest $\downarrow \beta$-galactosidase activity $\uparrow$
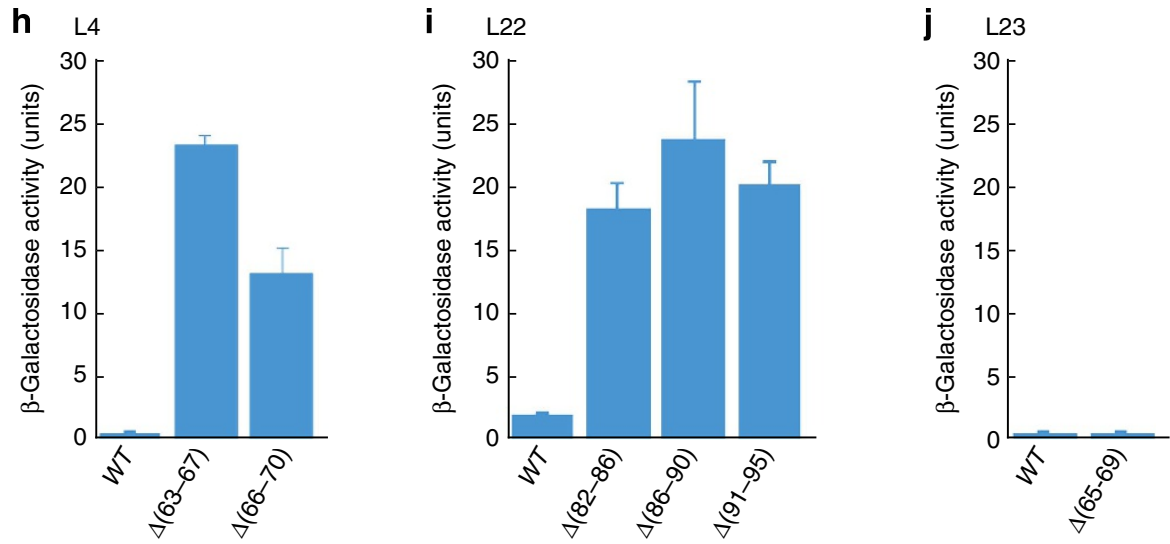

Figure 3 | Interactions of the MifM nascent chain with components of the ribosomal tunnel. (a-f) Contacts between MifM nascent chain (green) and the ribosome in the (a-c) upper and (d-f) lower region of the ribosomal tunnel. Electron density (mesh) is coloured for MifM (green), L4 (cyan), L22 (orange) and rRNA (grey). In (d-f) the cryo-EM map was filtered to $4 \AA$ resolution and the nascent chain modelled as a backbone trace. ( $(\mathbf{g}$ ) Schematic for the GFP-MifM-LacZ reporter used to monitor translational arrest, where stalling prevents $\beta$-galactosidase production in $B$. subtilis in vivo.

$(\mathbf{h}-\mathbf{j}) \beta$-galactosidase activity from the GFP-MifM-LacZ reporter (as in $\mathbf{g}$ ) expressed in B. subtilis strains bearing wildtype (WT) or truncated versions of ribosomal proteins (h) L4, (i) L22 or (j) L23. Error bars indicate the s.d. of three independent biological replicates.

number of amino-acid differences between B. subtilis and E. coli (Fig. 4b-d), suggesting that the specificity of MifM stalling may be conferred by L22. To test this possibility, we utilized the GFPMifM-LacZ reporter assay and demonstrated that replacing the $\beta$-hairpin of $B$. subtilis L22 with corresponding sequence from E. coli L22 led to an increase in $\beta$-galactosidase levels (Fig. 4e), consistent with a reduction in stalling at the MifM-derived sequence. Strikingly, reverting a single amino acid $(\mathrm{K} 90 \mathrm{M})$ in the $\beta$-hairpin of L22 from the E. coli sequence (K90) back to B. subtilis (M90) was sufficient to restore the low levels of $\beta$-galactosidase activity indicative of efficient stalling at MifM, whereas other reversions, such as the IM to FR double mutation at positions $85-86$ of $\mathrm{L} 22$ retained the elevated $\beta$-galactosidase levels (Fig. 4e). These findings suggest that the nature of the amino acid at position 90 of L22 can markedly influence the efficiency and specificity of stalling. Therefore, we introduced all 19 amino-acid substitutions at position 90 of B. subtilis L22 and monitored the $\beta$-galactosidase activity using the GFP-MifMLacZ reporter (Fig. 4f). A wide range of $\beta$-galactosidase activities was observed, with $\mathrm{K} 90$, as present in E. coli L22, producing the highest levels, whereas hydrophobic or aromatic amino acids, such as F, Y, L and I, in position 90 exhibited $\beta$-galactosidase activities similar to the wildtype L22 with M90 (Fig. 4f). While $\gamma$-proteobacteria, such as E. coli usually have K90 and most 
a

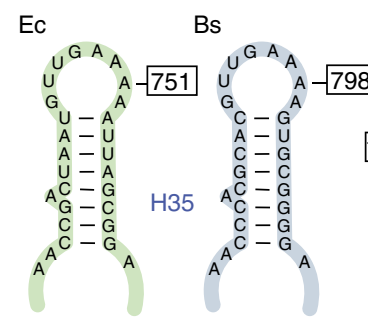

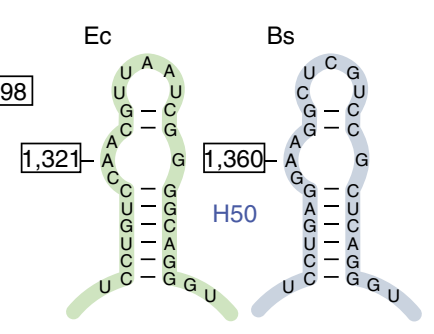

Ec b

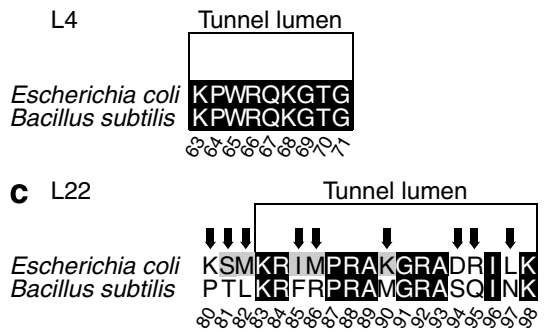

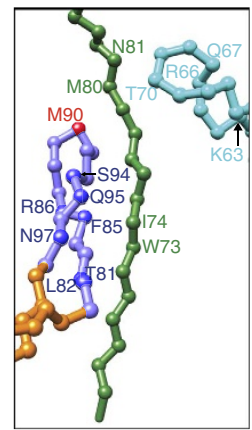

e

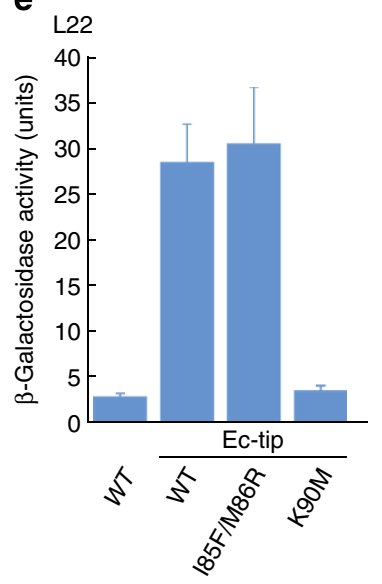

f

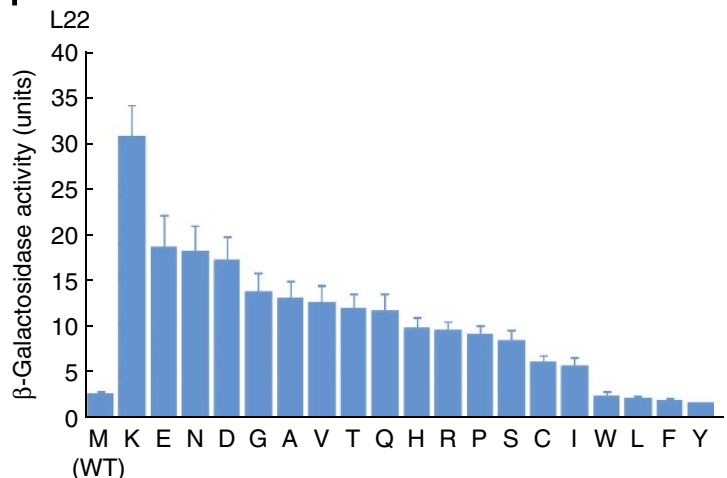

g

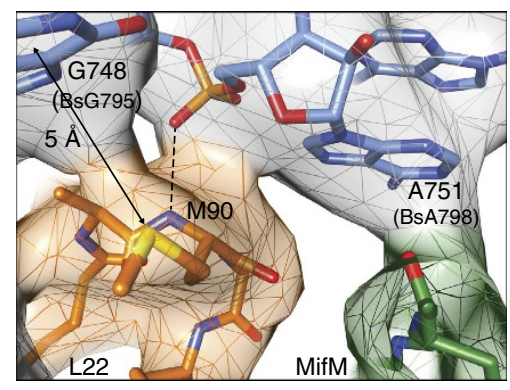

Figure 4 | Residue M90 in L22 contributes to the species specificity of MifM stalling. (a-c) Conservation between B. subtilis and E. coli of (a) $23 S$ rRNA nucleotides A751 (A798 in B. subtilis) and A1321 (A1360 in B. subtilis) within helices H35 and H50, respectively, as well as the tunnel lumen region of ribosomal proteins (b) L4 and (c) L22. In (b,c) similar and identical residues are shaded grey and black, respectively. (d) Overview of relative positions of MifM to tunnel lumen residues of L4 and L22. (e,f) $\beta$-galactosidase activity from the GFP-MifM-LacZ reporter (as in Fig. 3g) expressed in B. subtilis strains bearing wildtype (WT) L22 compared with (e) Ec-tip mutants where B. subtilis residues 80-98 are substituted with the equivalent $E$. coli residues (see d) and then additionally reverted to B. subtilis residues by substitutions I85F/M86R or K90M, or (f) L22 mutants where all possible amino-acid substitutions at position M90 of B. subtilis L22 were generated. Error bars indicate the s.d. of three independent biological replicates. (g) Interaction between the side chain of M90 of L22 with the base of G748 and potential hydrogen bonding (dashed line) between the backbone of M90 and the phosphate-oxygen of A751.

Bacillus species have M90, other bacterial lineages generally contain positively charged amino acids such as $\mathrm{R}, \mathrm{Q}, \mathrm{K}$ or $\mathrm{H}$ at the equivalent position (Supplementary Fig. 10), and are therefore unlikely to promote efficient MifM stalling.

M90 of L22 does not directly contact MifM but rather forms a Met-Sulfur aromatic interaction with G748 of the 23S rRNA (Fig. 4g), suggesting that the influence of this residue on MifM stalling may be indirect. One possibility is that M90 influences the interaction of MifM with nucleotide A751, either indirectly through G748, or more directly since the backbone nitrogen of M90 is within hydrogen bonding distance of the phosphateoxygen of A751 (Fig. 4g). In fact, the entire tip of the $\beta$-hairpin of L22 forms an intricate network of interactions with the 750-loop of $23 \mathrm{~S}$ rRNA helix H35 (Supplementary Fig. 11), suggesting that M90 mutations which alter the interaction with G748 as well as the conformation of the L22 tip could lead to rearrangements in the A750-loop. While the A751 region is structurally similar between B. subtilis and E. coli, the resolution does not allow us to exclude subtle changes or shifts in the positions of the nucleotides that could influence interaction with the MifM nascent chain. In addition, M90 mutations could alter the conformation of the $\beta$ hairpin of L22 and thereby influence the interaction between MifM and F85 of L22 (Fig. 3f). Support for the interplay between the $\beta$-hairpin of L22 and MifM residues located below the constriction comes from rescue experiments: Random mutagenesis of the $\beta$-hairpin (positions $80-89$ and 91-98) of B. subtilis L22-M90K was performed to search for second site mutations that restore MifM stalling and induce expression of a YidC2'-LacZ fusion, which led to the identification of mutations at position G91 of L22 that restored induction by up to $\sim 70 \%$ of the wildtype level, as well as lesser effects at positions R92, A93 and F85 (Supplementary Fig. 12). Second site mutations that could restore MifM stalling and induce expression of a YidC2' LacZ fusion in the presence of B. subtilis L22-M90K were also identified within the MifM nascent chain. In this latter case, random mutagenesis was performed at MifM positions 72-81, leading to the identification of the T72R mutation that restored induction by up to $\sim 60 \%$ of the wildtype level, with lesser effects also being observed at positions R75, K76 and F78 (Supplementary Fig. 12).

MifM inactivation of the PTC. To understand how the interaction of the MifM nascent chain with components of exit tunnel prevents stable binding of the A-tRNA and therefore leads to inhibition of peptide bond formation, we compared the conformation of nucleotides at the PTC in different states of peptide bond formation ${ }^{24-27}$ with the PTC of the MifM-SRC (Fig. 5a-c). U2584 and U2585 shift by $2-3 \AA$ on A-tRNA accommodation, that is, when moving from the unaccommodated (uninduced) to the accommodated (induced) state ${ }^{24-26}$ (Fig. 5a). In the MifM- 

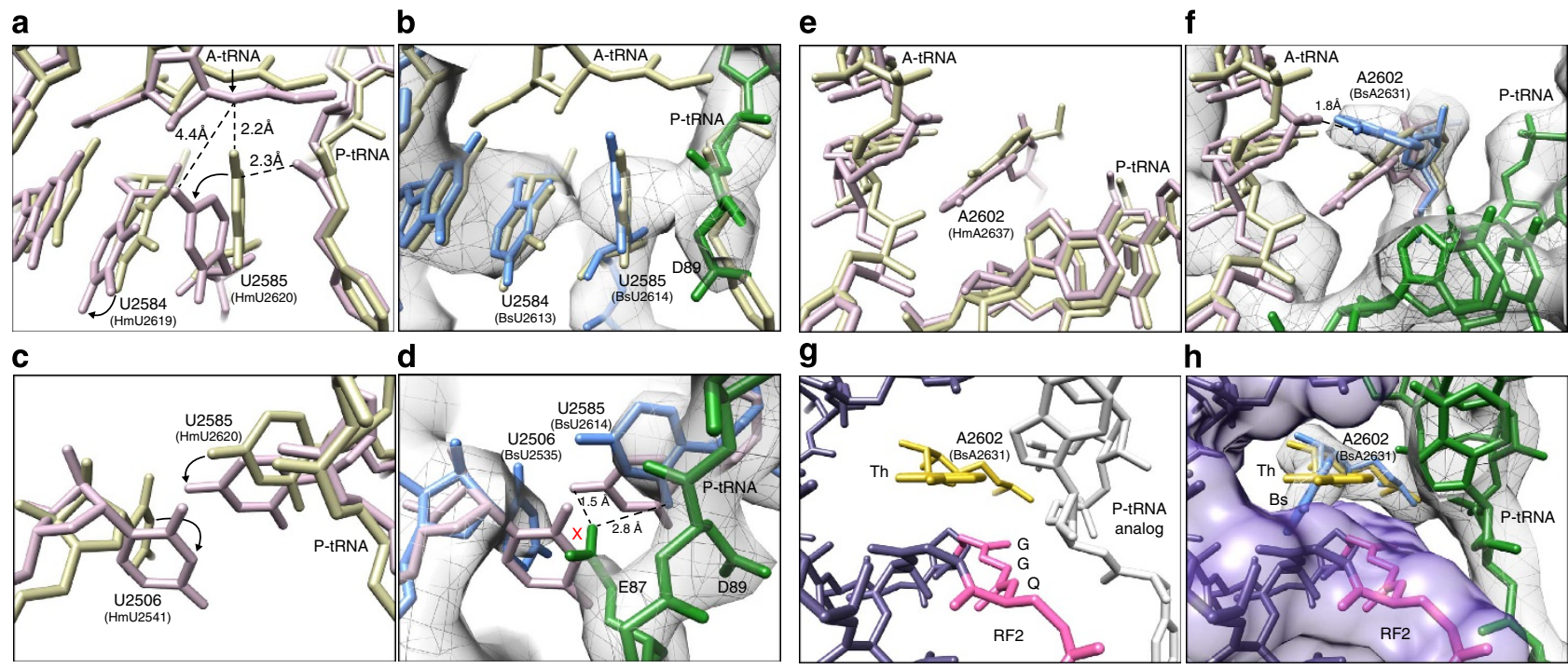

Figure 5 | MifM stabilizes the uninduced state of the PTC to inhibit A-tRNA accommodation. (a) A-tRNA accommodation leads to conformational changes in U2584 and U2585 shifting the PTC from an uninduced (yellow) to an induced state (salmon) ${ }^{24-26}$. (b) The PTC of the MifM-SRC (grey density with rRNA (blue) and P-tRNA (green)) resembles that of the uninduced state (yellow). (c) Rotation of U2506 is required to accommodate the shift in U2585 that occurs on A-tRNA accommodation 24-26. (d) E87 of MifM nascent chain (green) occupies the position of the induced state of U2506. (e) A-tRNA accommodation leads to a slight shift in the position of A2602. (f) The position of A2602 (blue) in the MifM-SRC encroaches on the A-tRNA binding site and is distinct from the position of A2602 in the uninduced (yellow) or induced states (pink) ${ }^{24-26}$. (g) A distinct conformation of A2602 (yellow) is required to position the GGQ motif of RF2 at the PTC to catalyse peptidyl-tRNA hydrolysis ${ }^{29-32}$. (h) The position of A2602 (blue) in the MifM-SRC is incompatible with the canonical binding position of RF2 at the PTC $29-32$.

SRC, U2584 and U2585 resemble the uninduced state, consistent with the absence of A-tRNA (Fig. 5b). The shift in U2585 that occurs during A-tRNA accommodation also requires a corresponding rotation of U2506 (Fig. 5c). In the MifM-SRC, the side chain of E87 overlaps in position with the induced conformation of U2506 (Fig. 5d), suggesting that the MifM nascent chain prevents A-tRNA accommodation by sterically preventing PTC nucleotides U2506, and thereby also U2585, from adopting their induced conformational states. E87 comprises part of the DEED sequence that is critical for MifM stalling ${ }^{2}$, however, single mutation of any of the amino acids within the DEED motif, including $\mathrm{E} 87 \mathrm{~A}$, does not markedly reduce stalling ${ }^{6}$. To completely abolish stalling, multiple $\mathrm{D} / \mathrm{E}$ to A mutations within this motif are required ${ }^{6}$, indicating some functional redundancy. One could speculate that within the context of the E87A mutation the role of E87 in blocking the U2506 shift is assumed by a neighbouring $\mathrm{D} / \mathrm{E}$ amino acids, however, without determining structures of the individual mutant MifM-RNCs it would be difficult to address this question.

In addition, during normal translation A2602 undergoes a slight shift on A-tRNA accommodation (Fig. 5e), whereas in the MifM-SRC, A2602 appears to adopt a defined position (Fig. 5f), which would restrict the entry of the incoming aminoacyl-tRNA to the A-site of the PTC. The conformation of the PTC of the MifM-SRC also provides an explanation as to why the MifMstalled ribosomes are resistant to the action of the RF1 and RF2 when the A90 position is a stop codon ${ }^{6,10}$ : Peptidyl-tRNA hydrolysis by RF1 and RF2 requires the accurate placement of the GGQ motif in domain 3 at the PTC of the ribosome, which induces specific conformations of PTC nucleotides, such as A2602 $28-32$ (Fig. 5g). This conformational change would be incompatible with the uninduced state of the PTC observed in the MifM-SRC (Fig. 5h). Indeed, the conformation of A2602 in the MifM-SRC is similar to that observed in the cryo-EM structure of the TnaC-SRC ${ }^{33}$, where translational arrest occurs because RF2dependent peptidyl-tRNA hydrolysis is inhibited ${ }^{7}$.
Although our structure does not provide direct insight into the mechanism of PTC inhibition when the ribosome stalls at subsequent downstream residues, we note that the SD-like helix formed between the MifM mRNA and the $5^{\prime}$ end of the $16 \mathrm{~S}$ rRNA (Fig. 2) is likely to contribute to multisite stalling, as SDlike sequences within the open reading frames of mRNAs have been reported to induce translational pausing in bacteria ${ }^{34}$. However, the introduction of frameshifts within the MifM leader peptide abolished translational stalling at all sites ${ }^{10}$, indicating that the SD-like helix alone is insufficient to induce pausing independently of the MifM nascent chain-induced stall, possibly because the helix cannot form in the absence of stalling or that SD-dependent pausing is actually negligible ${ }^{35}$.

\section{Discussion}

Collectively, our biochemical and structural findings lead us to propose a model for the mechanism and specificity of MifM stalling (Fig. 6): Residue M90 of L22 contributes to the species specificity of stalling, either through structural constraint given to A751 or residue F85 of L22. In the absence of any obvious relay of conformational changes to the PTC through the rRNA, we propose that these interactions, together with contacts between MifM and A1321 in 23S rRNA helix H50 and R66 of L4, promote a defined conformation of the MifM nascent chain such that residue E87 of MifM interacts with U2506 and U2585, thereby stabilizing the uninduced state of the PTC and preventing accommodation of the incoming A-tRNA (Fig. 6). In addition, we envisage that the pulling force exerted on the MifM nascent chain by interaction of the MifM TM with the YidC1/2 translocon during membrane insertion would release the MifM-dependent stalling by disrupting interactions between MifM and the ribosomal tunnel as well as displacing the E87 side chain of MifM and thus allowing U2506 and U2585 to adopt the induced state of the PTC that is necessary for aminoacyl-tRNA accommodation at the A-site. The mechanism of ligand- 


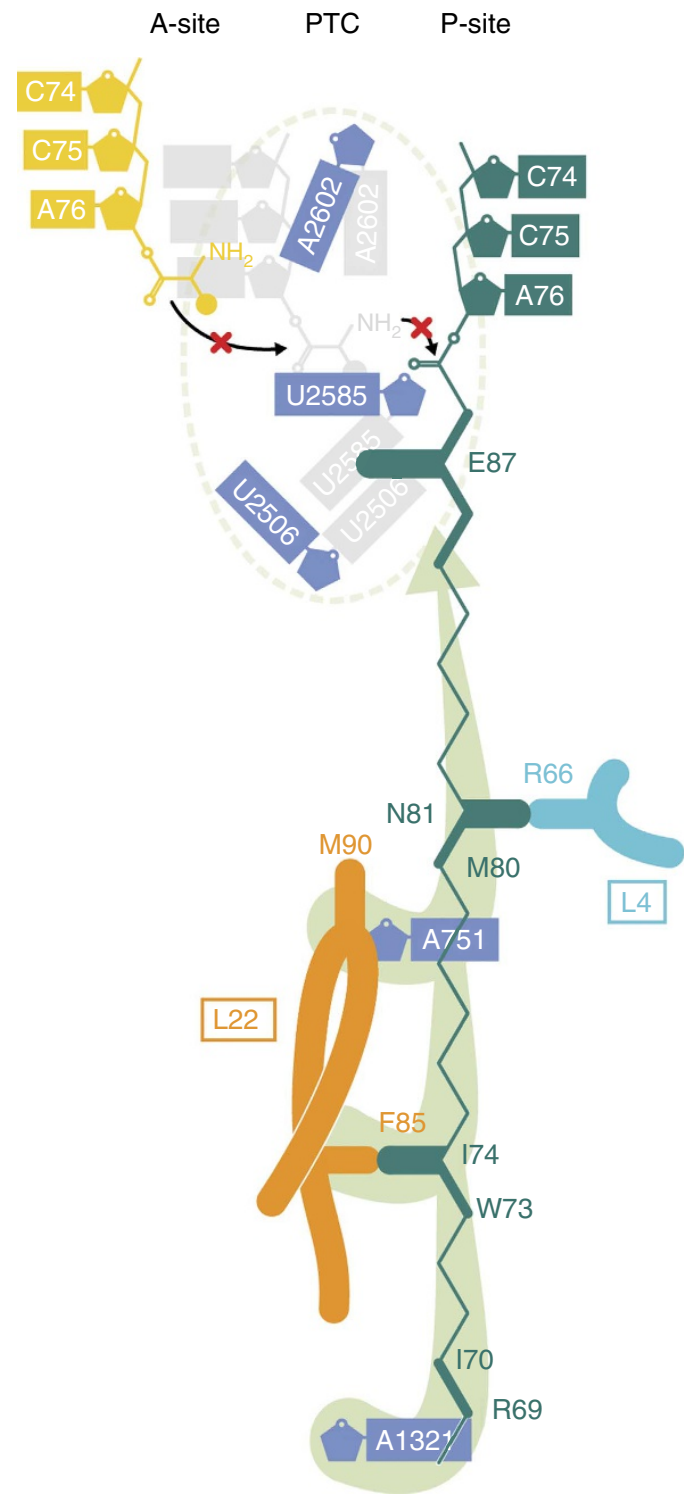

Figure 6 | Molecular basis for the specificity and mechanism of MifM-dependent translation arrest. Model illustrating how interactions of MifM within the ribosomal tunnel stabilize a specific conformation of E87 that prevents conversion of the uninduced state to the induced state and thereby blocks aminoacyl-tRNA binding at the A-site of the PTC.

independent translation arrest by MifM as described here is structurally distinct from previously characterized peptidedependent stalling systems, such as $\mathrm{Erm}^{36,37}$, TnaC ${ }^{33,38}$ and SecM ${ }^{18}$, illustrating the plasticity of the ribosome to employ diverse mechanisms to sense the nascent chain in the tunnel and evoke silencing of the PTC.

\footnotetext{
Methods

Strain and plasmid construction. B. subtilis strains and plasmids used in this study are listed in Supplementary Tables 1 and 2, respectively. B. subtilis strains were derivatives of PY79 (wildtype) ${ }^{39}$ and constructed by transformation with either plasmid or purified B. subtilis chromosomal DNA. Plasmids were constructed by standard cloning methods, fusion PCR and site-directed mutagenesis ${ }^{40}$ using primers and template plasmids listed in Supplementary Tables 2 and 3. Constructions of plasmids pCH735, pCH835, pCH913, pCH1142 and pEB71 were described previously (references shown in Supplementary Table 2). Plasmid pCH1517 was constructed by cloning an SphI-BglII-digested PCR fragment amplified from pCH913 using primers SP26 and SP27 into the SphI-BamHI site of pCH735. Plasmid pCH1570 was constructed by cloning a
}

BamHI-SphI fragment of pCH1567 into pCH1142. Plasmid pCH1567 was constructed by cloning a XbaI-SphI-digested PCR fragment amplified from PY79 chromosomal DNA using primers SP66 and SP67 into pCH1557. Plasmid pCH1557 was constructed as follows. A PCR fragment amplified from PY79 chromosomal DNA using primers SP68 and SP69 and another PCR fragment amplified from pEB71 using primers SP70 and SP71 were fused by the following PCR using primers SP68 and SP72, digested with both BamHI and XbaI and then cloned into pUC118. Plasmid pCH1587 was constructed as follows. A PCR fragment amplified from PY79 chromosomal DNA using primers SP28 and SP29 and another PCR fragment amplified from pEB71 using primers SP30 and SP31 were fused by the following PCR using primers SP28 and SP31, digested with both SacI and SphI and then cloned into pCH1142. Plasmid pCH1901 was constructed as follows. A PCR fragment amplified from pCH1587 using primers SP34 and SP35 and another PCR fragment amplified from pCH1587 using primers SP36 and SP37 were fused by the following PCR using primers SP34 and SP37, digested with both $\mathrm{XhoI}$ and SphI and then cloned into pCH1590. Other plasmids were constructed by site-directed mutagenesis using primers and templates shown in Supplementary Tables 2 and 3 .

Bacillus subtilis S12 translation extract. The B. subtilis S12 translation extract was prepared following a procedure described for E. coli S12 translation extract ${ }^{11,42}$, with some modifications. Briefly, cells (B. subtilis strain 168) were grown to $\mathrm{OD}_{600} 4.5$ in an 'INFORCE HT minifors' bench-top fermenter in $2 \times$ YPTG medium ( $16 \mathrm{gl}^{-1}$ peptone, $10 \mathrm{gl}^{-1}$ yeast extract, $5 \mathrm{gl}^{-1} \mathrm{NaCl}, 22 \mathrm{mM}$ $\mathrm{NaH}_{2} \mathrm{PO}_{4}, 40 \mathrm{mM} \mathrm{Na}_{2} \mathrm{HPO}_{4}, 19.8 \mathrm{gl}^{-1}$ glucose (sterile filtered)), with extra glucose feeding (feed: 10) at $37^{\circ} \mathrm{C}$ while maintaining pH 7.0 and oxygen level (60\%). Cells were collected at $5,000 \times g$ at $4{ }^{\circ} \mathrm{C}$ for $15 \mathrm{~min}$ and subsequently washed $3 \times$ in cold Buffer A (10 mM Tris-acetate ( $\mathrm{pH} 8.2), 14 \mathrm{mM}$ magnesium acetate, $60 \mathrm{mM}$ potassium glutamate, $1 \mathrm{mM}$ dithiothreitol and $6 \mathrm{mM} 2$-mercaptoethanol). Cells were snap-frozen in liquid nitrogen and stored at $-80^{\circ} \mathrm{C}$. About $15 \mathrm{~g}$ of cells were thawed on ice, resuspended in $10 \mathrm{ml}$ of cold buffer B (buffer A without 2-mercaptoethanol) and broken open in an 'microfluidics model 110I lab homogenizer', $3 \times$ at 15,000 psi. The lysate was cleared subsequently at $12,000 \times g$ and $4{ }^{\circ} \mathrm{C}$ for $10 \mathrm{~min}$ and incubated for $30 \mathrm{~min}$ at $37^{\circ} \mathrm{C}$ in a water bath. The cell extract was aliquoted, snap frozen and stored at $-80^{\circ} \mathrm{C}$.

PCR and in vitro transcription. PCR reaction (Phusion Master Mix (NEB), $98^{\circ} \mathrm{C}$ $\left.5 \mathrm{~min}\left(98^{\circ} \mathrm{C} 5 \mathrm{~s} ; 52^{\circ} \mathrm{C} 5 \mathrm{~s} ; 72^{\circ} \mathrm{C} 20 \mathrm{~s}\right) 28 \times 72{ }^{\circ} \mathrm{C} 10 \mathrm{~s}, 12^{\circ} \mathrm{C}\right)$ was prepared with MifMfor and MifMrev (Supplementary Table 3 ) and template DNA containing the sequence: (5'-TAATACGACTCACTATAGGGCGAATTGGCGGAAGGCCGTCA AGGCCACGTGTCTTGTCCATTAATTAACGTTTAACTTTAAGAAGGAGATA TACCAATGGGTCATCACCATCACCATCACCATCACGATTACGATATTCC AACGACCCTGGAAGTTCTGTTCCAGGGACCCGGTACCATGTTTGTGGAA TCGATAAATGACGTTTTATTCTTAGTCGATTTTTTCACAATTATTCTTCCT GCTCTAACGGCAATCGGGATTGCATTCCTCTTACGGGAGTGCCGTGCGG GCGAGCAATGGAAATCAAAACGAACAGATGGGCCCTACCCATACGATG TTCCAGATTACGCTGACTTTCTTATTATTATATATCATCGCATTACAACT TGGATACGTAAAGTCTTCCGCATGAATTCGCCTGTGAACGATGAGGAAG ACGCCGGTTCTCTTCTTTTATAA-3'; underlined are the T7 promoter region, ribosomal binding site, start codon, $8 \times$ His-tag and stop codon, respectively). PCR product was purified via spin columns and in vitro transcription reaction was set up using $1 \mu \mathrm{g}$ PCR product per $50 \mu \mathrm{l}$ reaction volume and T7 RNA polymerase. RNA was purified by $\mathrm{LiCl} /$ ethanol precipitation.

Preparation of the MifM-SRC. A volume of $1,360 \mu$ l of reaction mix containing $240 \mathrm{mM}$ HEPES-KOH (pH 8.2), $60 \mathrm{mM}$ glucose, $1.2 \mathrm{mM} \mathrm{ATP,} 0.85 \mathrm{mM} \mathrm{CTP}$, GTP, and UTP, $2 \mathrm{mM} \mathrm{DTT}, 0.17 \mathrm{mg} \mathrm{ml}^{-1}$ E. coli total tRNA mixture (from strain MRE600), $90 \mathrm{mM}$ potassium glutamate, $80 \mathrm{mM}$ of ammonium acetate, $8 \mathrm{mM}$ magnesium acetate, $20 \mathrm{mM}$ potassium phosphate dibasic ( $\mathrm{pH} 7.2), 34 \mu \mathrm{g} \mathrm{ml}^{-1}$ L-5-formyl-5, 6, 7, 8-tetrahydrofolic acid (folinic acid), $2.1 \mathrm{mM}$ (each) amino-acid mix, $2 \mathrm{mM}$ cysteine, $2 \%$ (w/v) PEG 8000 was preheated to $30^{\circ} \mathrm{C}$ before the addition of $540 \mu \mathrm{l}(27 \%(\mathrm{v} / \mathrm{v}))$ cell extract (S12 extract). The whole reaction was incubated at $30^{\circ} \mathrm{C}$ for 2 min before the addition of $100 \mu \mathrm{l}$ mifM mRNA (Supplementary Fig. 1) and further incubated at $30^{\circ} \mathrm{C}$ for $33 \mathrm{~min}$, shaking at 1,000 r.p.m.

Purification of the MifM-SRC. In vitro translation reactions $(4 \times 500 \mu \mathrm{l})$ were loaded onto $500 \mu \mathrm{l}$ sucrose cushion $(750 \mathrm{mM}$ sucrose) in Buffer C (50 mM HEPES, $250 \mathrm{mM}$ KOAc, $10 \mathrm{mM} \mathrm{MgOAc}, 0.1 \%$ DDM, 1/1,000 complete protease inhibitor (Roche), $0.2 \mathrm{U} \mathrm{ml}^{-1}$ RNasin, $\mathrm{pH} 7.2$ at $\left.4^{\circ} \mathrm{C}\right)$ and centrifuged for $150 \mathrm{~min}(45,000$ r.p.m., $4^{\circ} \mathrm{C}$ ) in a Beckman Coulter TLA 120.2 fixed-angle rotor. Pellets were resuspended in $4 \times 300 \mu \mathrm{l} 250 \mathrm{mM}$ sucrose in ice cold buffer $\mathrm{C}$ and loaded onto a Talon metal affinity chromatography column $(750 \mu \mathrm{l}$ resin) pre-equilibrated in $10 \mathrm{ml}$ buffer $\mathrm{C}$ containing $10 \mu \mathrm{g} \mathrm{\mu l}^{-1}$ bulk tRNA. The column was washed with $25 \mathrm{ml}$ buffer $\mathrm{C}$ until no significant absorption $\left(\mathrm{A}_{260}\right)$ could be detected in the wash fractions. The MifM-SRC, bound to the Talon matrix by MifM's $\mathrm{N}$-terminal $8 \times$ His-tag, was eluted in $4 \times 500 \mu \mathrm{l}$ buffer $\mathrm{C}$ containing $150 \mathrm{mM}$ Imidazole. The eluate was loaded onto $10-40 \%$ sucrose gradients (prepared with buffer C) and centrifuged for $13 \mathrm{~h}$ in a Beckman coulter SW40 Ti swinging bucket rotor $\left(20,000\right.$ r.p.m., $\left.4^{\circ} \mathrm{C}\right)$. Gradients were separated on a Biocomp Gradient Station ip 
and fractions containing 70S ribosomal particles were collected and pelleted for $3 \mathrm{~h}$ in a Beckman Coulter TLA 120.2 fixed-angle rotor $\left(45,000\right.$ r.p.m., $\left.4^{\circ} \mathrm{C}\right)$. The MifM-SRC pellet was resuspended in $28 \mu \mathrm{l} 30 \mathrm{mM}$ sucrose in buffer $\mathrm{C}$, on ice $\left(69 \mathrm{OD} \mathrm{ml}^{-1}\right)$, aliquoted and snap-frozen. Samples were further analysed by SDS-PAGE and western blotting.

MifM-SRC/SRP sample and cryogrid preparation. For grid preparation $2.5 \mu \mathrm{l}$ in vitro reconstituted B. subtilis signal recognition particle $\left(20 \mathrm{pmol}^{-1} \mathrm{l}^{-1}\right.$ in SRP buffer: $25 \mathrm{mM}$ HEPES, $100 \mathrm{mM}$ KOAc, $10 \mathrm{mM} \mathrm{MgOAc}, 1 \mathrm{mM}$ DTT, 10\% Glycerol, pH 7.2 at $\left.4{ }^{\circ} \mathrm{C}\right)$ were diluted to a final volume of $44.2 \mu \mathrm{l}$ with buffer $\mathrm{E}(50 \mathrm{mM}$ HEPES, $250 \mathrm{mM}$ KOAc, $2 \mathrm{mM}$ 2-mercaptoethanol, $0.06 \%$ Nikkol, pH 7.2 at $4{ }^{\circ} \mathrm{C}$ ) and activated by incubation at $30^{\circ} \mathrm{C}$ for $10 \mathrm{~min}$. Subsequently, $5.8 \mathrm{\mu l}(9.6 \mathrm{pmol})$ of MifM-SRC was added and incubated for $10 \mathrm{~min}$ at $30^{\circ} \mathrm{C}$. Finally the prepared mix was diluted with $22.5 \mu \mathrm{l}$ buffer F (50 mM HEPES, $250 \mathrm{mM} \mathrm{KOAc,} 2 \mathrm{mM}$ 2-mercaptoethanol, $\mathrm{pH} 7.2$ at $4{ }^{\circ} \mathrm{C}$ ) for optimal grid coverage.

Cryoelectron microscopy and single particle reconstruction. The MifM-SRC/ SRP sample was applied to $2 \mathrm{~nm}$ precoated Quantifoil R3/3 holey carbon supported grids and vitrified using a Vitrobot Mark IV (FEI Company). Data collection was performed using the EPU software (FEI) at NeCEN (Leiden, Netherlands) on a Titan Krios transmission electron microscope (FEI, Eindhoven, Netherlands) equipped with a Falcon II direct electron detector. Data were collected with the microscope set to $300 \mathrm{kV}$, a magnification of $125,085 \mathrm{x}$ (pixel size: $1.108 \AA$ ) in a defocus range of $1.0-2.5 \mu \mathrm{m}$ (Table 1). The data were provided as a series of seven frames (grid was pre-exposed for $55 \mathrm{~ms}, 4 \mathrm{e}^{-} \AA^{-2}$ before recording; dose per recorded frame: $8 \mathrm{e}^{-} \AA^{-2}$, exposure-time/ recorded frame: $110 \mathrm{~ms}$ ). Frames 1-3 (accumulated dose of $28 \mathrm{e}^{-} \AA^{-2}$ ) were summed after alignment using Motion Correction software ${ }^{43}$. Images were processed using a frequency-limited refinement protocol that helps prevent over-fitting ${ }^{44}$, specifically by truncation of high frequencies (in this case at $8 \AA$ ). As reported and expected ${ }^{44}$, we found that using this processing regime the 0.143 Fourier Shell Correlation (FSC) value provides a good indicator for the true average resolution of the map (Supplementary Fig. 2). In addition, the local resolution of the final map was calculated using ResMap ${ }^{19}$. Power spectra and defocus values were determined using the SPIDER TF ED command and recorded images were manually inspected for good areas and power-spectra quality. Data were processed further using the SPIDER software package ${ }^{45}$, in combination with an automated workflow as described previously ${ }^{46}$. After initial, automated particle selection based on the program SIGNATURE ${ }^{47}$, initial alignment was performed with 529,488 particles, using an E. coli 70 S ribosome as reference structure ${ }^{36}$. After removal of noisy particles $(101,109$ particles; 19\%), the data set was further sorted using an incremental K-means-like method of unsupervised 3D sorting ${ }^{48}$. Particles lacking density for P-tRNA $(75,984$ particles; $14 \%$ ) and those that did not improve in resolution during refinement were omitted from the data set. The major subpopulation (305,045 particles; $58 \%)$ showed the presence of stoichiometric densities for P-tRNA and could be refined to an average resolution of $3.9 \AA(0.143$ FSC) and a local resolution extending to $3.5 \AA$ for the core of the ribosome as computed using ResMap ${ }^{19}$ (Supplementary Fig. 2) and was used for modelling. Subsequently, this main volume could be sorted further into a subvolume containing the signal recognition particle bound to the ribosome (158,726 particles; $30 \%$ ), which will be described elsewhere (Beckert et al., unpublished). The final volumes were subjected to the program EM-BFACTOR ${ }^{49}$ in order to apply an automatically determined negative B-factor for sharpening of the map.

Molecular modelling and map-docking procedures. The B. subtilis 5S, $16 \mathrm{~S}$ and 23S rRNA sequences were taken from GeneBank; Gene ID 2914271, 936774 and 939981, respectively. Structure-based sequence alignments were generated using Sequence to Structure $(\mathrm{S} 2 \mathrm{~S})^{50}$ based on X-ray structure derived models of the small and large ribosomal subunit of $E$. coli (PDB ID 4KIX/Y) $)^{51}$. Molecular dynamics flexible fitting $(\mathrm{MDFF})^{52}$ in $\mathrm{VMD}^{53}$ was used for initial fitting and refinement of the rRNA models into the electron density. The resulting models of the B. subtilis rRNAs were manually inspected and adjusted according to features of the electrondensity using $\operatorname{Coot}^{54}$, followed by refinement using $\operatorname{erraser}^{55}$ and the real-space refine tool in PHENIX ${ }^{56}$ (Table 1). A total of 29 of the 32 large subunit proteins (L1, L7/L12 and L9 were excluded) from the B. subtilis 50S subunit were generated using the homology with the equivalent $E$. coli and T. thermophilus protein templates (PDB codes 4KIX and 3I8I, respectively). The models were initially fitted to the density using Chimera ${ }^{57}$, followed by real-space refinement using PHENIX ${ }^{56}$ (Table 1). For the 19 proteins of the B. subtilis 30S subunit, homology models were generated based on protein templates from $T$. thermophilus (PDB code $3 \mathrm{~V} 2 \mathrm{C}$ ) using HHPred ${ }^{58}$ and fitted to the density using Chimera ${ }^{57}$ and refined in Coot $^{54}$. The resolution of the core of the large subunit ( $3.5 \AA$, Supplementary Fig. 2) enabled the majority of the sidechains for the large ribosomal proteins to be modelled (Supplementary Fig. 3), whereas the lower resolution of the core of the small subunit (4.0 $\AA$, Supplementary Fig. 2) limited the molecular modelling to the backbone of the small subunit ribosomal proteins. The fit of the atomic models was validated as described ${ }^{59}$ by calculating the FSC between a theoretical density derived from the atomic models for the B. subtilis 70 S ribosome (generated using the CP FROM PDB SPIDER command) and the unsharpened cryo-EM map of
MifM-SRC (Supplementary Fig. 4). The volumes were multiplied (using the MU SPIDER command) with a 'soft' mask generated from the theoretical model density (using the TH M and FQ NP SPIDER commands) previous to calculating their Fourier shell correlation (using the RF 3 SPIDER command).

Figure preparation. Figures showing electron densities and atomic models were generated using UCSF Chimera ${ }^{57}$.

及-galactosidase assays. B. subtilis cells were cultured at $37^{\circ} \mathrm{C}$ in $\mathrm{LB}$ media for $\beta$-galactosidase activity assay ${ }^{60}$. Cells were collected from cultures of $\mathrm{OD}_{600}=$ $0.5-1.0$. To test $\beta$-galactosidase activities on agar plates, cells were grown on Difco sporulation medium (DSM) agar plates containing $60 \mathrm{\mu g} \mathrm{ml}^{-1}$ 5-bromo-4-chloro3 -indolyl- $\beta$-D-galactoside (X-gal) at $37^{\circ} \mathrm{C}^{6}$.

Isolation of suppressor mutants. Plasmid library for isolation of intragenic suppressor mutants of the $r p l V(M 90 K)$ mutant were prepared as follows. The second point mutation was introduced into either of the 80-89th or the 91st-98th codons of $r p l V(M 90 K)$ on the original plasmid $\mathrm{pCH} 1897$ by site-directed mutagenesis using random mixed mutagenic primers listed in Supplementary Table 3 (SP38-SP55). B. subtilis strain SCB3378 (gfp-mif $M^{35-95}$-yidC2'-lacZR cat, rplV94) was transformed with the mutagenized plasmid library and then blue colonies were isolated on DSM agar plates including $3 \mu \mathrm{g} \mathrm{ml}^{-1}$ kanamycin and $60 \mu \mathrm{g} \mathrm{ml}{ }^{-1} \mathrm{X}$-gal at $37^{\circ} \mathrm{C}$. The original strain SCB3378 harbours the rplV94 mutation, which has a seven codon insertion within the $r p l V$, resulting in arrest-defective phenotype. Strain SCB3378 was made from SCB824 (gfp-mif $M^{35-95}-y i d C 2^{\prime}-$ lacZ $\left.2 c a t, r p l V^{+}\right)$ by isolating a spontaneous erythromycin resistant mutant. Suppressor mifM mutants were isolated as follows: Plasmid library was prepared by site-directed mutagenesis using template plasmid pCH835 (gfp-mif $M^{35-95}$-yidC2'-lacZRcat) and random mixed mutagenic primers listed in Supplementary Table 3 (SP56-65) that are designed to introduce a point mutation into either one of the 72nd-81st codons of mifM. B. subtilis strain SCB3348 ( $r p l V(M 90 K))$ was transformed with the mutagenized plasmid library and then blue colonies were isolated on DSMchloramphenicol-X-gal agar plates at $37^{\circ} \mathrm{C}$

\section{References}

1. Voorhees, R. M. \& Ramakrishnan, V. Structural basis of the translational elongation cycle. Annu. Rev. Biochem. 82, 203-236 (2013).

2. Wen, J. D. et al. Following translation by single ribosomes one codon at a time Nature 452, 598-603 (2008).

3. Peil, L. et al. Distinct XPPX sequence motifs induce ribosome stalling, which is rescued by the translation elongation factor EF-P. Proc. Natl Acad. Sci. USA 110, 15265-15270 (2013).

4. Starosta, A. L. et al. Translational stalling at polyproline stretches is modulated by the sequence context upstream of the stall site. Nucleic Acids Res. 42, 10711-10719 (2014).

5. Wilson, D. N. \& Beckmann, R. The ribosomal tunnel as a functional environment for nascent polypeptide folding and translational stalling. Curr. Opin. Struct. Biol. 21, 1-10 (2011).

6. Chiba, S. \& Ito, K. Multisite ribosomal stalling: a unique mode of regulatory nascent chain action revealed for MifM. Mol. Cell 47, 863-872 (2012).

7. Gong, F. \& Yanofsky, C. Instruction of translating ribosome by nascent peptide Science 297, 1864-1867 (2002).

8. Vazquez-Laslop, N., Thum, C. \& Mankin, A. S. Molecular mechanism of drugdependent ribosome stalling. Mol. Cell 30, 190-202 (2008).

9. Nakatogawa, H. \& Ito, K. The ribosomal exit tunnel functions as a discriminating gate. Cell 108, 629-636 (2002).

10. Chiba, S., Lamsa, A. \& Pogliano, K. A ribosome-nascent chain sensor of membrane protein biogenesis in Bacillus subtilis. EMBO J. 28, 3461-3475 (2009).

11. Chiba, S. et al. Recruitment of a species-specific translational arrest module to monitor different cellular processes. Proc. Natl Acad. Sci. USA 108, 6073-6078 (2011).

12. Dalbey, R. E., Wang, P. \& Kuhn, A. Assembly of bacterial inner membrane proteins. Annu. Rev. Biochem. 80, 161-187 (2011).

13. Butkus, M. E., Prundeanu, L. B. \& Oliver, D. B. Translocon 'pulling' of nascent SecM controls the duration of its translational pause and secretion-responsive secA regulation. J. Bacteriol. 185, 6719-6722 (2003).

14. Gumbart, J., Schreiner, E., Wilson, D. N., Beckmann, R. \& Schulten, K. Mechanisms of SecM-mediated stalling in the ribosome. Biophys. J. 103, 331-341 (2012).

15. Ismail, N., Hedman, R., Schiller, N. \& von Heijne, G. A biphasic pulling force acts on transmembrane helices during translocon-mediated membrane integration. Nat. Struct. Mol. Biol. 19, 1018-1022 (2012).

16. Chiba, S. \& Ito, K. MifM monitors total YidC activities of Bacillus subtilis including that of YidC2, the target of regulation. J. Bacteriol. 197, 99-107 (2014). 
17. Bhushan, S. et al. alpha-Helical nascent polypeptide chains visualized within distinct regions of the ribosomal exit tunnel. Nat. Struct. Mol. Biol. 17, 313-317 (2010).

18. Bhushan, S. et al. SecM-stalled ribosomes adopt an altered geometry at the peptidyltransferase center. PLoS Biol. 19, e1000581 (2011).

19. Kucukelbir, A., Sigworth, F. J. \& Tagare, H. D. Quantifying the local resolution of cryo-EM density maps. Nat. Methods 11, 63-65 (2014).

20. Schuwirth, B. et al. Structures of the bacterial ribosome at $3.5 \AA$ resolution. Science 310, 827-834 (2005).

21. Selmer, M. et al. Structure of the $70 \mathrm{~S}$ ribosome complexed with mRNA and tRNA. Science 313, 1935-1942 (2006).

22. Yusupova, G., Jenner, L., Rees, B., Moras, D. \& Yusupov, M. Structural basis for messenger RNA movement on the ribosome. Nature 444, 391-394 (2006).

23. Kaminishi, T. et al. A snapshot of the $30 \mathrm{~S}$ ribosomal subunit capturing mRNA via the Shine-Dalgarno interaction. Structure 15, 289-297 (2007).

24. Schmeing, T. M. et al. A pre-translocational intermediate in protein synthesis observed in crystals of enzymatically active 50 S subunits. Nat. Struct. Biol. 9, 225-230 (2002).

25. Schmeing, T. M., Huang, K. S., Kitchen, D. E., Strobel, S. A. \& Steitz, T. A. Structural insights into the roles of water and the 2' hydroxyl of the P site tRNA in the peptidyl transferase reaction. Mol. Cell 20, 437-448 (2005).

26. Schmeing, T. M., Huang, K. S., Strobel, S. A. \& Steitz, T. A. An induced-fit mechanism to promote peptide bond formation and exclude hydrolysis of peptidyl-tRNA. Nature 438, 520-524 (2005).

27. Voorhees, R. M., Weixlbaumer, A., Loakes, D., Kelley, A. C. \& Ramakrishnan, V. Insights into substrate stabilization from snapshots of the peptidyl transferase center of the intact 70 S ribosome. Nat. Struct. Mol. Biol. 16, 528-533 (2009)

28. Polacek, N. et al. The critical role of the universally conserved A2602 of $23 \mathrm{~S}$ ribosomal RNA in the release of the nascent peptide during translation termination. Mol. Cell 11, 103-112 (2003).

29. Weixlbaumer, A. et al. Insights into translational termination from the structure of RF2 bound to the ribosome. Science 322, 953-956 (2008).

30. Laurberg, M. et al. Structural basis for translation termination on the $70 \mathrm{~S}$ ribosome. Nature 454, 852-857 (2008).

31. Korostelev, A. et al. Crystal structure of a translation termination complex formed with release factor RF2. Proc. Natl Acad. Sci. USA 105, 19684-19689 (2008).

32. Korostelev, A. A. Structural aspects of translation termination on the ribosome. RNA 17, 1409-1421 (2011).

33. Seidelt, B. et al. Structural insight into nascent polypeptide chain-mediated translational stalling. Science 326, 1412-1415 (2009).

34. Li, G. W., Oh, E. \& Weissman, J. S. The anti-Shine-Dalgarno sequence drives translational pausing and codon choice in bacteria. Nature 484, 538-541 (2012).

35. Borg, A. \& Ehrenberg, M. Determinants of the rate of mRNA translocation in bacterial protein synthesis. J. Mol. Biol. (2014).

36. Arenz, S. et al. Molecular basis for erythromycin-dependent ribosome stalling during translation of the ErmBL leader peptide. Nat. Commun. 5, 3501 (2014).

37. Arenz, S. et al. Drug Sensing by the ribosome induces translational arrest via active site perturbation. Mol. Cell 56, 446-452 (2014).

38. Bischoff, L., Berninghausen, O. \& Beckmann, R. Molecular basis for the ribosome functioning as an L-tryptophan sensor. Cell Rep. 9, 469-475 (2014).

39. Youngman, P., Perkins, J. B. \& Losick, R. A novel method for the rapid cloning in Escherichia coli of Bacillus subtilis chromosomal DNA adjacent to Tn917 insertions. Mol. Gen. Genet. 195, 424-433 (1984).

40. Sawano, A. \& Miyawaki, A. Directed evolution of green fluorescent protein by a new versatile PCR strategy for site-directed and semi-random mutagenesis. Nucleic Acids Res. 28, E78 (2000).

41. Kim, T. W. et al. Simple procedures for the construction of a robust and costeffective cell-free protein synthesis system. J. Biotechnol. 126, 554-561 (2006).

42. Kim, T.-W., Kim, H.-C., Oh, I.-S. \& Kim, D.-M. A highly efficient and economical cell-free protein synthesis system using the S12 extract of Escherichia coli. Biotechnol. Bioproc. E. 13, 464-469 (2009).

43. Li, X. et al. Electron counting and beam-induced motion correction enable near-atomic-resolution single-particle cryo-EM. Nat. Methods 10, 584-590 (2013).

44. Scheres, S. H. \& Chen, S. Prevention of overfitting in cryo-EM structure determination. Nat. Methods 9, 853-854 (2012).

45. Frank, J. et al. SPIDER and WEB: processing and visualization of images in 3D electron microscopy and related fields. J. Struct. Biol. 116, 190-199 (1996)

46. Becker, T. et al. Structural basis of highly conserved ribosome recycling in eukaryotes and archaea. Nature 482, 501-506 (2012).

47. Chen, J. Z. \& Grigorieff, N. SIGNATURE: a single-particle selection system for molecular electron microscopy. J. Struct. Biol. 157, 168-173 (2007).

48. Loerke, J., Giesebrecht, J. \& Spahn, C. M. Multiparticle cryo-EM of ribosomes. Methods Enzymol. 483, 161-177 (2010).
49. Fernandez, J. J., Luque, D., Caston, J. R. \& Carrascosa, J. L. Sharpening high resolution information in single particle electron cryomicroscopy. J. Struct. Biol. 164, 170-175 (2008).

50. Jossinet, F. \& Westhof, E. Sequence to Structure (S2S): display, manipulate and interconnect RNA data from sequence to structure. Bioinformatics 21, 3320-3321 (2005).

51. Pulk, A. \& Cate, J. H. Control of ribosomal subunit rotation by elongation factor G. Science 340, 1235970 (2013).

52. Trabuco, L. G., Villa, E., Mitra, K., Frank, J. \& Schulten, K. Flexible fitting of atomic structures into electron microscopy maps using molecular dynamics. Structure. 16, 673-683 (2008).

53. Humphrey, W., Dalke, A. \& Schulten, K. VMD-visual molecular dynamics. J. Mol. Graph. 14, 33-38 (1996).

54. Emsley, P. \& Cowtan, K. Coot: model-building tools for molecular graphics. Acta Crystallogr. D Biol. Crystallogr. 60, 2126-2132 (2004).

55. Chou, F. C., Sripakdeevong, P., Dibrov, S. M., Hermann, T. \& Das, R. Correcting pervasive errors in RNA crystallography through enumerative structure prediction. Nat. Methods 10, 74-76 (2013).

56. Adams, P. D. et al. PHENIX: a comprehensive Python-based system for macromolecular structure solution. Acta Crystallogr. D Biol. Crystallogr. 66, 213-221 (2010).

57. Pettersen, E. F. et al. UCSF chimera; a visualization system for exploratory research and analysis. J. Comput. Chem. 25, 1605-1612 (2004).

58. Hildebrand, A., Remmert, M., Biegert, A. \& Soding, J. Fast and accurate automatic structure prediction with HHpred. Proteins 77(Suppl 9): 128-132 (2009).

59. Chen, S. et al. High-resolution noise substitution to measure overfitting and validate resolution in $3 \mathrm{D}$ structure determination by single particle electron cryomicroscopy. Ultramicroscopy 135, 24-35 (2013).

60. Rubio, A., Jiang, X. \& Pogliano, K. Localization of translocation complex components in Bacillus subtilis: enrichment of the signal recognition particle receptor at early sporulation septa. J. Bacteriol. 187, 5000-5002 (2005).

\section{Acknowledgements}

This research was supported by grants from the Deutsche Forschungsgemeinschaft (WI3285/3-1 to D.W.; FOR1805 and GRK1721 to D.N.W. and R.B.), European Research Council (to R.B.), MEXT and JSPS Grant-in-Aid for Scientific Research (20523517, 25291006, 26116008 to S.C. and 20247020 to K.I.), the Private University Strategic Research Foundation Support Program from MEXT (S1101029 to K.I.), the Fondation pour la Recherche Médicale (AJE201133 to C.A.I) and the Conseil Régional d'Aquitaine (2012 1301009 to C.A.I) and the European Union (PCIG14-GA-2013-631479 to C.A.I) We thank Rishi Matadeen and Sacha DeCarlo for EM data collection, Naoka Kagatsume and Shiori Yamamoto for preliminary data collection, Tomoe Takino and Chika Tsutsumi, for technical support and Andreas Anger for help with molecular modelling of the 30 S subunit.

\section{Author contributions}

D.S. prepared translation extract, MifM-SRC complex performed single-particle cryo-EM analysis and model building. S.C. and N.S.-C. performed in vivo Bacillus studies. D.S. and A.I. performed the model refinement and validation. O.B. helped with sample preparation. All authors interpreted the data and helped with the manuscript preparation. D.S. and D.N.W. wrote the manuscript.

\section{Additional information}

Accession codes. The cryo-EM map and associated atomic coordinates have been deposited in the EMDB and PDB with the accession numbers EMD-6306 and PDB ID 3J9W, respectively.

Supplementary Information accompanies this paper at http://www.nature.com/ naturecommunications

Competing financial interests: The authors declare no competing financial interests.

Reprints and permission information is available online at http://npg.nature.com/ reprintsandpermissions/

How to cite this article: Sohmen, D. et al. Structure of the Bacillus subtilis $70 \mathrm{~S}$ ribosome reveals the basis for species-specific stalling. Nat. Commun. 6:6941 doi: $10.1038 /$ ncomms7941 (2015).

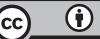

This work is licensed under a Creative Commons Attribution 4.0 International License. The images or other third party material in this article are included in the article's Creative Commons license, unless indicated otherwise in the credit line; if the material is not included under the Creative Commons license, users will need to obtain permission from the license holder to reproduce the material. To view a copy of this license, visit http://creativecommons.org/licenses/by/4.0/ 